\title{
Temporal order and tactile patterns
}

\author{
JAMES C. CRAIG \\ Indiana University, Bloomington, Indiana \\ and \\ XU BAIHUA \\ Hangzhou University, Hangzhou, Zhejiang, People's Republic of China
}

\begin{abstract}
Temporal order judgments (TOJs) were obtained for tactile stimuli presented to subjects' fingerpads. In one set of measurements, pairs of spatial patterns were presented successively to a single fingerpad (same-site condition), to two fingers on the same hand (ipsilateral condition), or to two fingers on opposite hands (bilateral condition). The subjects were instructed to report which one of the two patterns was presented first. TOJs were more accurate in the same-site condition than in either the ipsilateral or the bilateral conditions. In the ipsilateral and bilateral conditions, performance improved when judging which one of two locations received a stimulus first, although performance levels were still lower than in the same-site condition. Increasing the size of the pattern set from which the two patterns to be judged were drawn had only a slight effect on samesite performance and no effect on ipsilateral/bilateral performance; however, changing the nature of the patterns had a considerable effect on same-site performance and a smaller effect on ipsilateral/bilateral performance. Introducing an intensity imbalance between members of the pair of stimuli also had a large effect on same-site TOJs: a less intense stimulus tended to be judged as being presented first. In the bilateral condition, however, there was a small effect in the reverse direction: more intense stimuli tended to be judged as being presented first. The intensity imbalance had no effect in the ipsilateral condition. The results suggest that different mechanisms are responsible for TOJs for patterns presented to the same-site and to separate sites and, furthermore, that separate sites may constitute separate channels for spatial information.
\end{abstract}

Previous studies of temporal order for tactile stimuli have investigated subjects' accuracy in reporting which one of two locations on the skin was stimulated first (Hill, 1971; Hill \& Bliss, 1968; Hirsh \& Sherrick, 1961; Marks et al., 1982; Sherrick, 1970; Taylor, 1978). In the present study, we investigated the ability of subjects to judge the temporal order of tactile patterns. The impetus for this study was threefold. First, much of the information that we obtain by means of the sense of touch comes from the sequential examination of surfaces. In many instances, the stimuli received by the skin are spatial patterns. Searching for a particular coin in your pocket, locating the correct light switch in the dark, or reading braille all involve sequential processing of spatial patterns. The identification of objects or the obtaining of information by the sense of touch may require individuals to perceive correctly the temporal order in which particular spatial patterns are encountered. Also, Sherrick (1970) has pointed out the im-

This research was supponted by National Institutes of Health Grant NS09783. The authors thank Roger Rhodes for his assistance in these experiments. The experiments were carried out while the second author was a visiting research scholar at Indiana University as part of the Indiana University/Hangzhou University exchange. The first author's address is: James C. Craig, Department of Psychology, Indiana University, Bloomington, IN 47405. The second author's address is: Xu Baihua, Department of Psychology, Hangzhou University, Hangzhou, Zhejiang, People's Republic of China. portance of understanding temporal order when matching the design of haptic displays to the capabilities of the skin.

A second impetus for the present study was a series of studies in which the identifiability of one spatial pattern was examined in the presence of a second, temporally adjacent spatial pattern. The presence of one pattern often interferes with, or "masks," the identification of the other, target pattern (Craig, 1985b; Craig \& Evans, 1987). The pattern interfering with the target, the masker, has been shown to have a number of effects on the perception of the target. The masker may increase the number of features that the target appears to contain (Evans \& Craig, 1986), it may alter the apparent location of the target's features (Craig, 1989), or it may be responded to as though it were the target-that is, subjects may respond with the masker rather than with the target (Evans, 1987a, 1987b). This latter type of error could be the result of the close temporal proximity of two patterns producing problems in judging temporal order. The subject often has to judge which one of the two patterns was presented first and which second. Thus, it is not surprising that on some occasions the wrong pattern may be given as a response. To increase our understanding of temporal masking requires a greater understanding of the factors that affect temporal order judgments (TOJs).

A third impetus for the present study is provided by the question of how the skin combines information from the same site as compared to separate sites of stimula- 
tion. Previous studies have examined how subjects combined spatial patterns presented to the same or to separate sites on the hand (Craig, 1983, 1985a, 1985b). In one set of measurements, patterns were divided in half, and either half the pattern was presented to one finger and the other half to another finger, or both halves were presented to the same finger. The subjects were able to identify a pattern better when the two halves of the pattern were delivered to fingers on opposite hands, bilaterally, rather than to fingers on the same hand, ipsilaterally, and nearly as well as when the two halves were delivered to the same finger. These results indicated that the subjects had some difficulty attending to two patterns presented simultaneously (or nearly simultaneously) to two fingers on the same hand, but less difficulty in attending to two fingers on opposite hands (Craig, 1985a). These results also indicated that stimuli presented to the same site were combined at an earlier stage of processing than were stimuli presented to separate sites. Studies of temporal masking of spatial patterns have led to a similar conclusion. As noted before, two spatial patterns delivered to the same location can mask one another. When the stimuli are delivered to adjacent locations, either ipsilaterally (Craig, 1983) or bilaterally, as in recent measurements in our laboratory, little or no masking is seen. Measurements of TOJs provide additional information on how spatial patterns are processed when presented to the same site and to separate sites.

Temporal order has been studied for relatively simple tactile stimuli. Hirsh and Sherrick (1961) presented mechanical taps to the two index fingers. The subject's task was to report which location received the tap first. The now classic finding was that approximately $20 \mathrm{msec}$ was required between onsets of successive taps to the skin for subjects to report correctly the temporal order on $75 \%$ of the trials.

Subsequently, Sherrick (1970) studied temporal order for tactile stimuli delivered to two locations on the same thigh and to two locations on opposite thighs. The bilateral condition yielded a threshold value nearly identical to that obtained earlier by Hirsh and Sherrick (1961)-20 msecwhereas the ipsilateral condition produced a larger threshold. Even larger thresholds have been obtained with electrocutaneous stimuli applied to several locations on the forehead and abdomen (Marks et al., 1982).

In the above studies, TOJs were based on the location of the stimuli. The stimuli themselves were identical. In a study involving the temporal ordering of three stimuli, Taylor (1978) examined more complex stimuli. In his study, stimuli could differ from one another in terms of the locations to which they were presented, in terms of frequency, or, in some conditions, in terms of both location and frequency. Performance improved when frequency and location provided redundant information for TOJs.

In the present study, we examined accuracy in TOJs when subjects were required to base their decision on the spatial form of a pattern. Using only form as a cue, in
Experiment 1, we examined TOJs for patterns presented to the same location-the fingerpad-and for patterns presented to two fingerpads on the same hand and on opposite hands. We also examined TOJs when the subjects could base their decisions on the location of the pattern. Superior performance in the same-site condition in Experiment 1 suggested that subjects might base their judgments of temporal order on cues unavailable in the separate-site conditions. To examine these possible cues, additional sets of patterns were tested in Experiment 2, and the relative intensity of the spatial patterns was manipulated in Experiment 3.

\section{EXPERIMENT 1}

To our knowledge, no previous studies have compared TOJs for tactile stimuli delivered to the same location with TOJs for tactile stimuli delivered to separate locations. Predicting performance in the same-site condition relative to ipsilateral and bilateral conditions is difficult. At brief stimulus-onset asynchronies (SOAs), two patterns presented to the same location may mask one another. However, in Experiment 1, patterns were presented at equal intensities and were relatively simple spatial patterns, lessening the likelihood that masking would interfere with the TOJ. Several studies (Craig, 1982; Evans, 1987a; Evans \& Craig, 1986) have obtained results that suggest that two patterns presented in close temporal and spatial contiguity may form a composite pattern through temporal integration. Evans (1987a, 1987b) hypothesized that elements of the trailing pattern are represented more strongly in the composite than are elements of the leading pattern. If such were the case in the present experiment, then performance at brief SOAs for the same-site condition might be improved either by the formation of composite patterns or by judging the relative intensity of the two patterns. Subjects may use cues not available when two patterns are presented to separate sites. In tasks involving the presentation of patterns to two separate sites, subjects in our laboratory did not report that the patterns formed a single composite of the sort formed with patterns presented to the same site. TOJs might therefore be expected to be less accurate than in same-site conditions.

There is a second reason to expect separate-site performance to be poorer than same-site performance. It may be that in the separate-site conditions, subjects will treat the temporal order task as though it consists of two parts, initially judging which of the two sites received a pattern first and then identifying which of the two patterns was presented to that site. The addition of the second task, pattern identification, might well increase the threshold for temporal order. In fact, the threshold might be greater than the values obtained by Hirsh and Sherrick (1961), whose subjects needed to judge only which location received a stimulus first.

Several investigators have noted that TOJs depend upon a number of procedural factors, such as the presence or absence of trial-by-trial feedback, the amount of practice 
subjects receive, and the number of presentations of a pair of stimuli a subject is given prior to making his or her response (Gengel \& Hirsh, 1970; Sternberg \& Knoll, 1973). Preliminary work in our laboratory suggested that the range of temporal separations presented to subjects, whether or not trials were blocked by SOA, and the kinds of instructions provided to subjects all affected the accuracy of subjects' judgments. The procedures we adopted were those that tended both to stabilize and to maximize performance levels.

\section{Method}

Subjects. The subjects in all three experiments were students employed by the laboratory. All subjects were experienced in tactile pattern-recognition tasks and in temporal order tasks.

Apparatus. The tactile stimuli were generated on displays interfaced with a PDP-11/34 computer. The computer controlled the presentation and timing of the stimuli and recorded the subjects' responses. Each tactile display was composed of 144 pins arranged in a 6-column $\times 24$-row array and was part of the Optacon (Bliss, Katcher, Rogers, \& Shepard, 1970), a reading aid for the blind. The array measured $1.1 \times 2.7 \mathrm{~cm}$ and fit against the distal portion of the subject's fingerpad. When activated, each pin in the array vibrated at $230 \mathrm{~Hz}$.

Stimuli. The stimuli consisted of two tactile patterns. One was a vertical pattern, generated by activating 2 columns of pins on the tactile array; the other was a horizontal pattern, generated by activating 3 rows of pins. Representations of the two patterns are shown in Figure 1. The number of pins activated for each pattern was the same, 18 , and the intensity was set at $36 \mathrm{~V}$, the voltage applied to each active pin in the array. The duration of each stimulus was 26 msec.

Procedure. The subjects were tested individually. On each trial, the vertical and horizontal patterns were presented in random order. Three conditions were examined. One was referred to as the "samesite condition," in which the two patterns were generated on one tactile array and presented to the subject's left index fingerpad. In the other two conditions, referred to as the "ipsilateral condition" and the "bilateral condition," two tactile arrays were used. For the ipsilateral condition, the subject's left middle fingerpad rested on one of the two tactile displays, and the subject's left index fingerpad rested on the other tactile display. For the bilateral condition, the subject's left middle fingerpad rested on one of the two displays, and the right index fingerpad rested on the other display. One of the two patterns was randomly selected and presented to one display and the other to the second display. In both the samesite and the separate-site conditions, the subject's task was to indicate which pattern was presented first.

Initially, three SOAs, 17, 35, and $70 \mathrm{msec}$, were tested. Trials were blocked by SOA, and the subjects were informed that this was the case. An experimental session consisted of nine blocks of 40 trials. During each session, one of the three conditions was tested for three successive blocks of trials. The SOAs were tested in random order. The subjects were informed that on half of the trials the vertical pattern would be presented first, and on half of the trials the horizontal pattern would be presented first. Each subject completed seven sessions, one session per day. Because performance was poor in the two separate-site conditions, an additional set of measurements was made that included longer SOAs of $17,35,70$, 140 , and $280 \mathrm{msec}$.

Separate-site measurements were also made in which the same pattern was presented to both sites, and the subjects judged which one of the sites received the stimulus first. Three SOAs were tested: 17,35 , and $70 \mathrm{msec}$. Bilateral and ipsilateral conditions were alternated, and each condition was tested for three blocks of 40 trials.

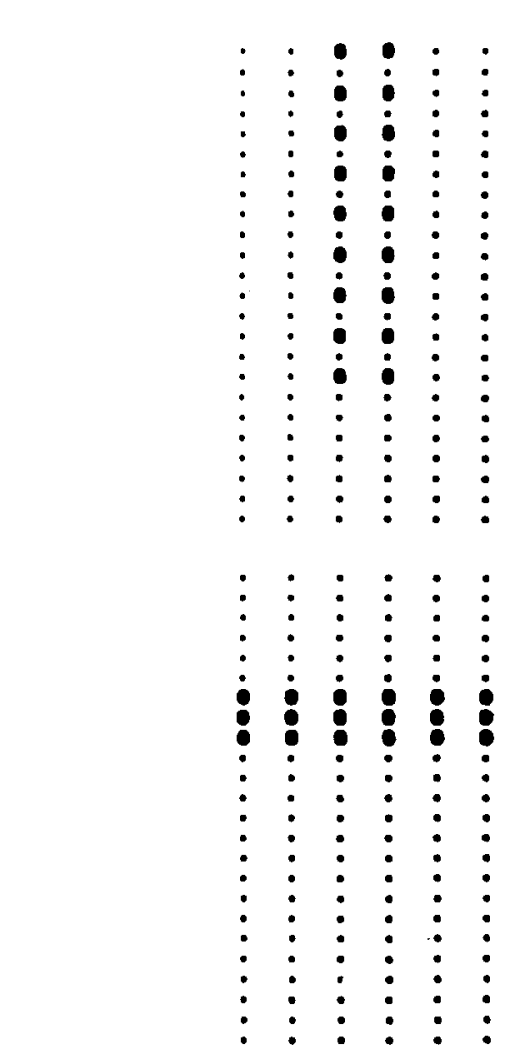

Figure 1. Representations of the two stimuli as they appeared on the tactile array.

In the same-site and ipsilateral conditions, the subjects responded after each trial by pressing one of two numbered buttons on a keyboard placed in front of them. In the bilateral condition, the subjects indicated their judgment by using foot pedals. A correct response was followed by the word "correct" appearing on a visual display in front of the subject. An incorrect response was followed by the word "incorrect." The subject wore earphones through which white noise was presented to reduce auditory cues produced by the tactile arrays.

In the course of preliminary measurements, the subjects mentioned a number of cues that they felt were useful in judging temporal order. In the ipsilateral and bilateral conditions, they mentioned the direction of apparent motion (Sherrick, 1968a, 1968b; Sherrick \& Rogers, 1966). They also observed that the stimulus that they believed was presented first felt more intense than the second stimulus. This observation was made more frequently in the bilateral than in the ipsilateral condition. For the same-site condition, the subjects mentioned that the second pattern seemed clearer than the first and possibly more intense. The subjects reported that when two patterns were presented to the same site with a brief temporal separation between them, often only a single pattern was perceived. The overall shape of this single pattern seemed to change depending upon which of the two simple patterns was presented first. All of these cues were discussed with the subjects prior to their participation in the experiments reported here.

\section{Results and Discussion}

The data were analyzed in several ways. First, the data were analyzed by subject. All subjects in this experiment showed similar functions, and the data were averaged across subjects. Second, the probability of responding 


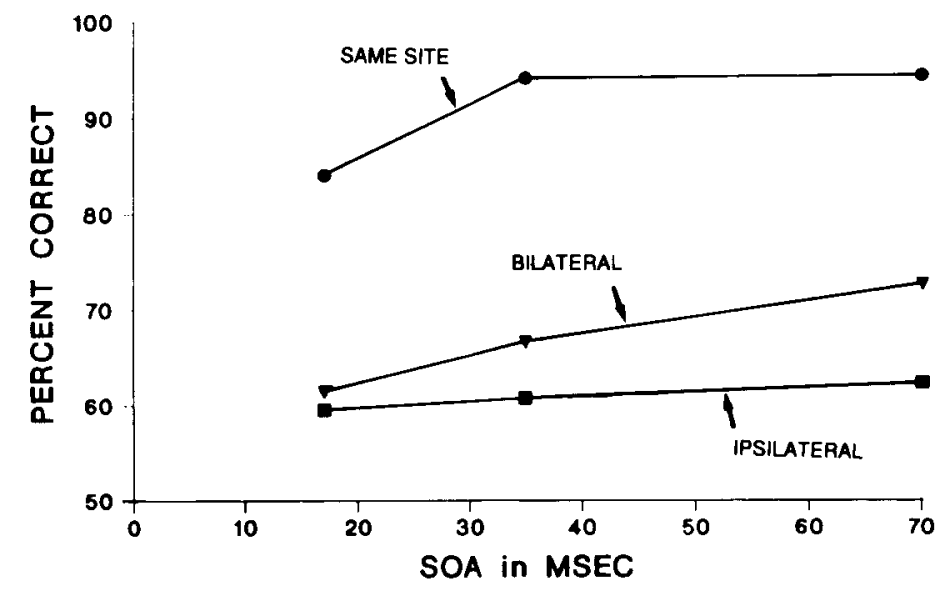

Figure 2. Percentage of correct responses in judging temporal order as a function of SOA. Stimuli were presented to the same site, to two sites ipsilaterally, or to two sites bilaterally. The subjects had to judge which one of two patterns was presented first.

"vertical" first was plotted as a function of SOA. The SOAs ranged from $-70 \mathrm{msec}$ (the horizontal pattern leading the vertical pattern by $70 \mathrm{msec}$ ) to $+70 \mathrm{msec}$ (the vertical pattern leading the horizontal by $70 \mathrm{msec}$ ). A psychometric function was fitted to these data using an approximation to the cumulative normal. A functionminimization routine called STEPIT (Chandler, 1970) was used to generate the psychometric functions. The functions for the same-site, ipsilateral, and bilateral conditions were nearly symmetrical about $0 \mathrm{msec}$ and showed a shift in the point of subjective simultaneity (PSS) of no more than $2 \mathrm{msec}$. The results with the second set of measurements using longer SOAs were treated in a similar fashion. Again, a small shift in PSS, 2 msec or less, was found. In these separate-site conditions, there was a small bias favoring the stimulus presented to the left fingerpad; however, for the purposes of these analyses, this bias was ignored.

Because the bias favoring one of the two patterns was small, the data were collapsed across the two different orders of pattern presentation. The data for the briefer SOAs are presented in Figure 2 as percentage of correct responses in judging which one of the two patterns was presented first. The data for the longer SOAs are presented in Figure 3. Each point in the two figures represents the mean performance of 4 subjects and is based on a total of 960 trials. The standard errors of the means, based on the total number of blocks of trials, ranged from $1 \%$ to $2 \%$. For the same-site and separate-site conditions, the first session was considered a practice session and is not included in the data analysis reported below. Using the mean and standard deviation calculated from the STEPIT

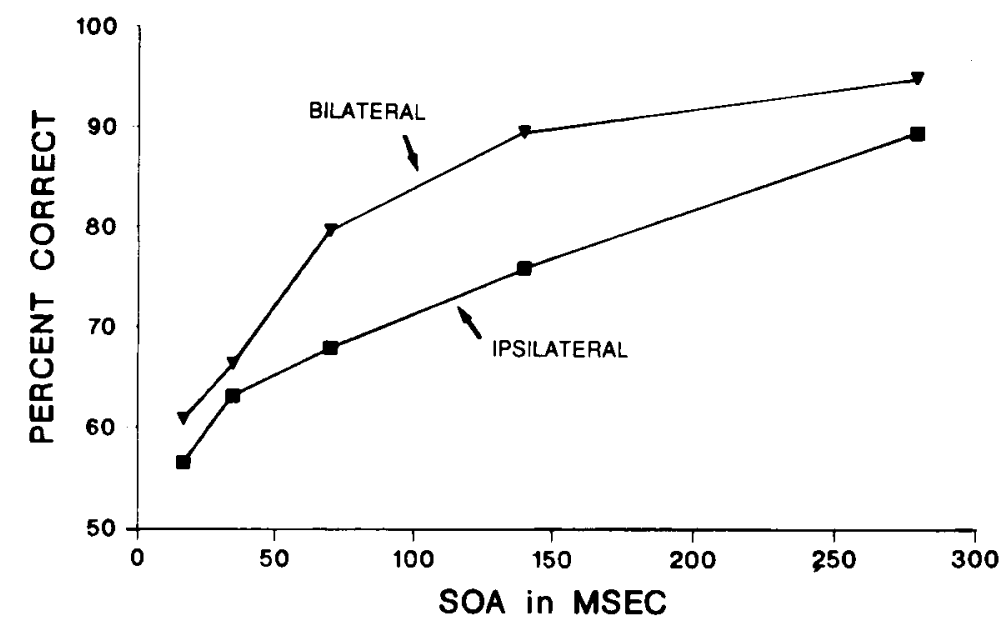

Figure 3. Percentage of correct responses in judging temporal order as a function of SOA. Similar to Figure 2, with longer SOAs tested. 
function, $25 \%$ and $75 \%$ performance levels were determined and a mean threshold-the SOA corresponding to 75\% correct performance-was calculated.

For all experiments, unless otherwise noted, the data were analyzed by means of a Wilcoxon matched-pairs signed-ranks test, two-tailed. The analysis confirmed what is apparent in Figure 2: performance in the same-site condition was significantly better than in either of the other two conditions. At all three SOAs, $p$ was less than .01 . The threshold in the same-site condition was $12 \mathrm{msec}$. The thresholds for the bilateral measurements, Figures 2 and 3 , were 68 and $62 \mathrm{msec}$, respectively, and for the ipsilateral conditions, 120 and $130 \mathrm{msec}$, respectively.

What accounts for the superior performance in the same-site condition? As noted in the introduction to Experiment 1 , additional cues seem to have been available in the same-site condition that were not available or as apparent in the separate-site conditions. The subjects reported that the relative intensity of the patterns changed with temporal position. They also reported that often at brief SOAs they did not feel two patterns as they did in the separate-site conditions but rather a single, composite pattern. If the subjects felt a composite pattern, they reported that the shape of this pattern appeared to change depending upon the temporal position of the two patterns making up the composite. The subjects could successfully report the temporal order by identifying which one of two composites they perceived.

Clearly, the subjects had considerably more difficulty in judging temporal order for patterns presented to separate sites than for patterns presented to the same site. In addition, in the two separate-site conditions, ipsilateral performance lagged behind bilateral performance. In Figure 2, bilateral performance is significantly better than ipsilateral performance at $70 \mathrm{msec}(p<.02)$, and in Figure 3, it is significantly better at 70,140 , and $280 \mathrm{msec}$ $(p<.02)$. The reason for the poorer ipsilateral performance may be the inability of subjects to attend as read- ily to ipsilateral stimulation as to bilateral stimulation (Craig, 1985a).

The results of our measurements in which subjects judged which one of two locations received a pattern first (same pattern delivered to both locations) are shown in Figure 4. Each point represents a total of 1,280 trials from 4 subjects. The thresholds for the ipsilateral and bilateral conditions are 19 and $20 \mathrm{msec}$, respectively, very similar to Hirsh and Sherrick's (1961) results obtained in a similar task. A comparison of the results in Figure 4 with those in Figures 2 and 3 shows the much longer temporal separations required for judging temporal order for patterns delivered to separate sites than for judging simply which location received a pattern first. TOJs for patterns presented to separate sites may be more difficult than comparable TOJs for location because in the former case, subjects must first judge the temporal order of the locations and then identify which pattern was presented to which location. An additional factor in disrupting TOJs for patterns may be the nature of the trials. In judging which one of two patterns came first, the patterns are not correlated with location, and there are four different types of trials: two orders of pattern presentation $x$ two locations. When judging location, subjects receive only two types of trials, which might simplify the task. The results of Experiment 2 bear, in part, on this issue because additional patterns were used in order to create several different types of trials.

\section{EXPERIMENT 2}

The results of Experiment 1 suggest that TOJs for patterns were more accurate in the same-site condition than in the separate-site condition because in the same-site condition, the subjects had additional cues from the interactions between the two patterns, cues that were not available in the separate-site condition. In Experiment 2, we examined some of the factors that might affect perfor-

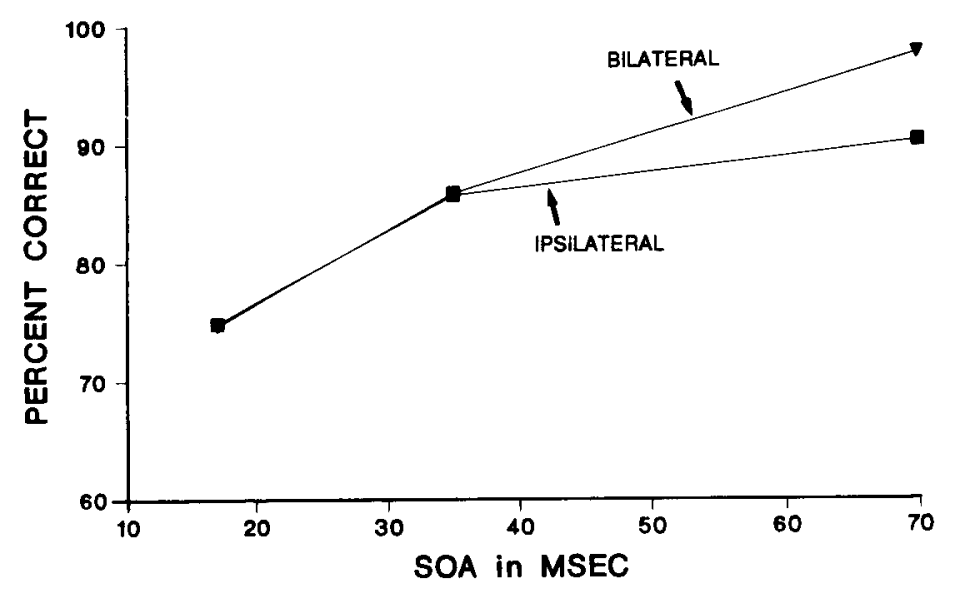

Figure 4. Percentage of correct responses in judging temporal order as a function of SOA. The subjects had to judge which one of two locations received a stimulus first. 
mance differentially in the same- and separate-site conditions. Two factors were examined: the number and the type of patterns. In all cases, subjects judged the temporal order of two patterns, but the number of patterns from which the two to be judged were drawn was increased. The number of patterns was increased for two reasons. First, if subjects use the shape of composite patterns in the same-site condition, then increasing the number of simple patterns to be judged from two to four (resulting in an increase in the number of possible composite patterns from 2 to 12 ) might cause difficulty in the samesite, relative to the separate-site, condition. Second, the conclusion from Experiment 1 that there is a consistent same-site advantage may be misleading if such an advantage is based on restricting the number of patterns to be judged.

To increase the number of patterns, a new set of patterns (Figure 5A) was generated. The results with these new patterns suggested that the nature of the patterns themselves affected performance in the same-site condition; therefore, a second set of patterns (Figure 5B) was generated. Both sets of patterns were used in both the same-site and the separate-site conditions.

\section{Method}

Procedure. The testing procedures were similar to those used in Experiment 1. Two different sets of patterns were used. The patterns, referred to as Set A and Set B, are shown in Figure 5.
The subjects were first tested with the four patterns in Set A. On each trial, the subjects were presented with two randomly selected patterns. As before, the subject's task was to select the pattern that had been presented first. The subjects pressed one of four buttons, or, in the bilateral condition, one of four foot pedals, and received trial-by-trial feedback. Four SOAs were tested: 35, 70, 140, and 245 msec.

In the first measurements with Set $A$, the subjects were presented with two of the four patterns but were not informed which two patterns would be presented. There was a possibility that the subjects might err in their TOJs because of an inability to identify the two patterns being presented. To eliminate this possibility, a second set of measurements was made in which the subjects were informed which two of the four patterns were to be presented. Any response other than one of the two patterns presented was not permitted. In addition, if subjects try to use composites as a basis for TOJs in the same-site condition, informing them which two patterns are to be presented may help them use the composites. In a third set of measurements, the subjects received extensive training in the samesite condition on two of the four patterns. In addition, another group of subjects, who had been tested only with the two patterns used in Experiment 1 (Figure 1), were tested repeatedly with two patterns selected from Set $A$. The second and third sets of measurements should replicate the same-site results seen in Figure 2, unless the particular patterns used affect TOJs.

A second group of 3 subjects were tested with patterns from Set B. Three SOAs were tested: 17,35 , and $70 \mathrm{msec}$. Initially, the subjects received the same two patterns repeatedly-Patterns 1 and 2 , Patterns 2 and 3, or Patterns 1 and 3-one pair for each of the 3 subjects. Following eight sessions with two patterns, the subjects were tested with three patterns-1,2, and 3 . The subjects were informed prior to each trial which two of the three patterns were to

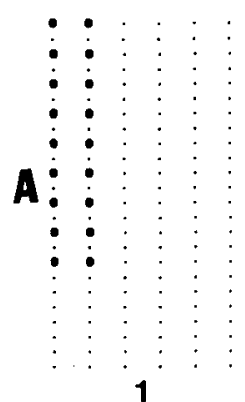

1

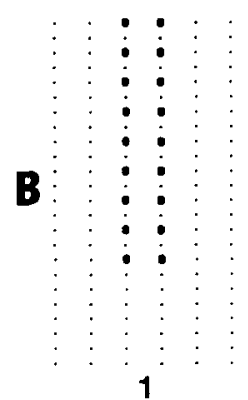

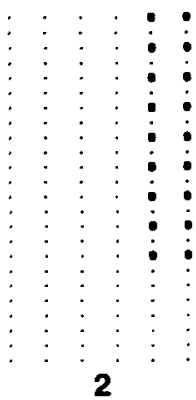

2

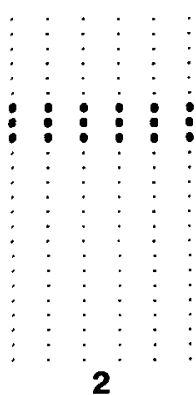

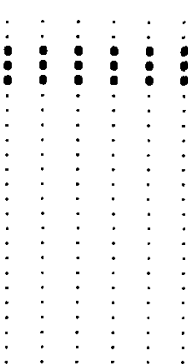

3

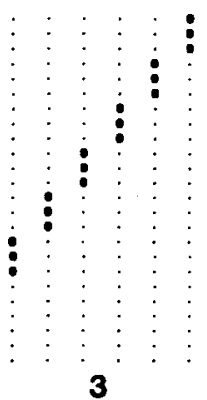

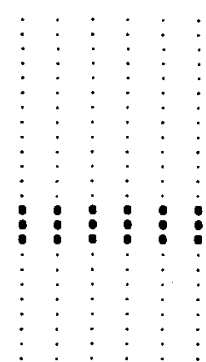

4

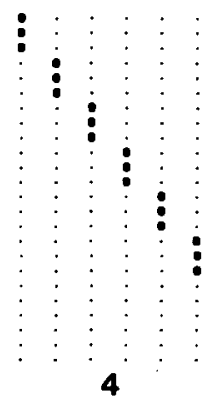

Figure 5. Representations of the two sets of patterns tested in Experiment 2. 
be presented, and only responses to one of those two patterns were permitted. The subjects were tested for five sessions and then began testing with all four patterns. Again, the subjects were informed in advance which two of the four patterns were to be presented. The subjects were tested for four sessions in this condition.

\section{Results and Discussion}

The results from the first series of measurements with patterns from Set A are shown in Figure 6. Each point represents a total of 960 trials from 4 subjects. Whereas previous measurements (see Figure 2) had shown samesite performance to be superior to separate-site performance, Figure 6 shows the reverse. The thresholds for the same-site, ipsilateral, and bilateral conditions were 100,74 , and $41 \mathrm{msec}$, respectively. The thresholds for the separate-site conditions were somewhat less than in Experiment 1, whereas the same-site threshold was more than eight times the comparable value in Experiment 1.

These results show that the stimulus manipulations involved in presenting subjects with the patterns in Set A produced a large deleterious effect on same-site performance and a relatively smaller effect - an improvementon separate-site performance. It is less clear what it is specifically about these stimulus manipulations that is responsible for the changes in performance. One possibility is that the subjects had difficulty in identifying the two patterns in the same-site condition. Previous studies have shown that the presentation of one stimulus in close temporal and spatial proximity (the same fingerpad) to a second stimulus may cause the second to be mislocalized (Craig, 1989). In the present experiment, such mislocalization might have caused the subjects to respond with one of the four patterns that had not been presented, resulting in poorer performance. Informing the subjects in advance which two patterns were to be presented should have reduced that problem. The results of the second set of measurements, in which the subjects were instructed about the patterns, are shown in Figure 7. Each point represents the results from 4 subjects, a total of 960 trials.
A comparison of the pre- and postinstruction functions shows that both ipsilateral and same-site performance improved somewhat with instructions, and that bilateral performance improved a little. Across the four SOAs, the increase in the percentage of correct responses was $8.2 \%$ for the same site, $7.5 \%$ for ipsilateral, and $1.8 \%$ for bilateral. The thresholds for the three conditions-same-site, ipsilateral, and bilateral-were 67,48 , and $36 \mathrm{msec}$, respectively. Providing information about which two of the four patterns was to be presented appears to have produced some improvement in performance; however, there was not enough improvement in the same-site condition to produce the superior performance seen in Experiment 1.

The relatively poor same-site performance suggests that the subjects were unable to use composite patterns as the basis for TOJs even when informed which two patterns were to be presented. The difficulty in forming and using composites may be due to the increased number of composites. It is also possible that there is something about the patterns in Set A that interferes with TOJs. Two results indicate that the latter was the case: the first result was from the subjects whose results are shown in Figure 7, who received additional testing with just two of the four patterns in the same-site condition only. The results from these subjects are shown in Figure 8 . The number of trials ranges from 480 to 940 per point. The reason same-site performance was poor when the subjects were tested with all four patterns from Set A (Figure 7) appears to be that several subjects had difficulty in making their TOJs with these particular patterns. A second result from the group of subjects who were tested only on two of the four patterns (i.e., had received no experience with all four patterns) also suggests that the nature of the patterns themselves may have interfered with the same-site TOJs. This group of subjects had even poorer performance than the original subjects, probably because they had less experience with the patterns. The 4 subjects showed similar levels of performance. Their mean threshold was

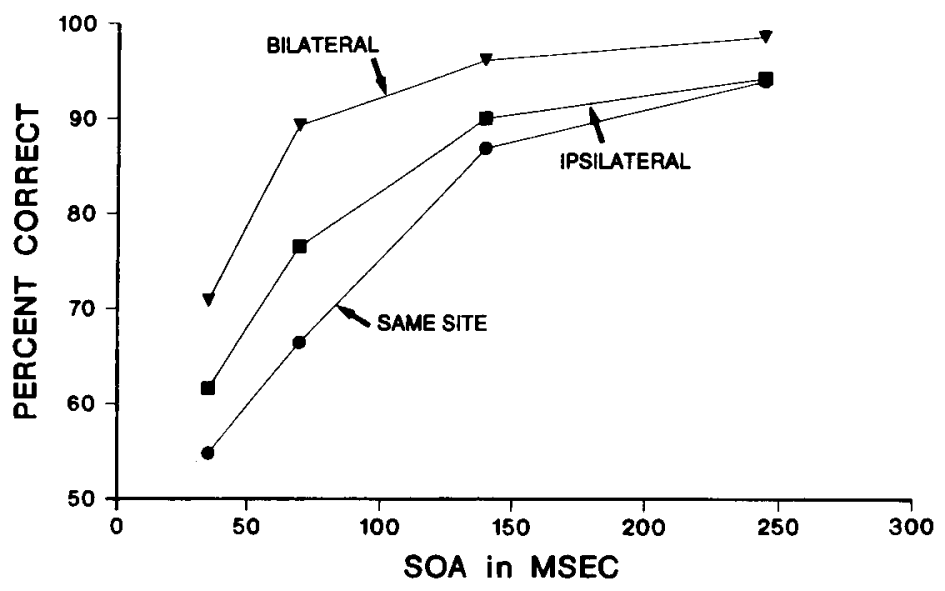

Figure 6. Percentage of correct responses in judging temporal order as a function of SOA. Patterns to be judged were selected from Pattern Set A, Figure 5. 

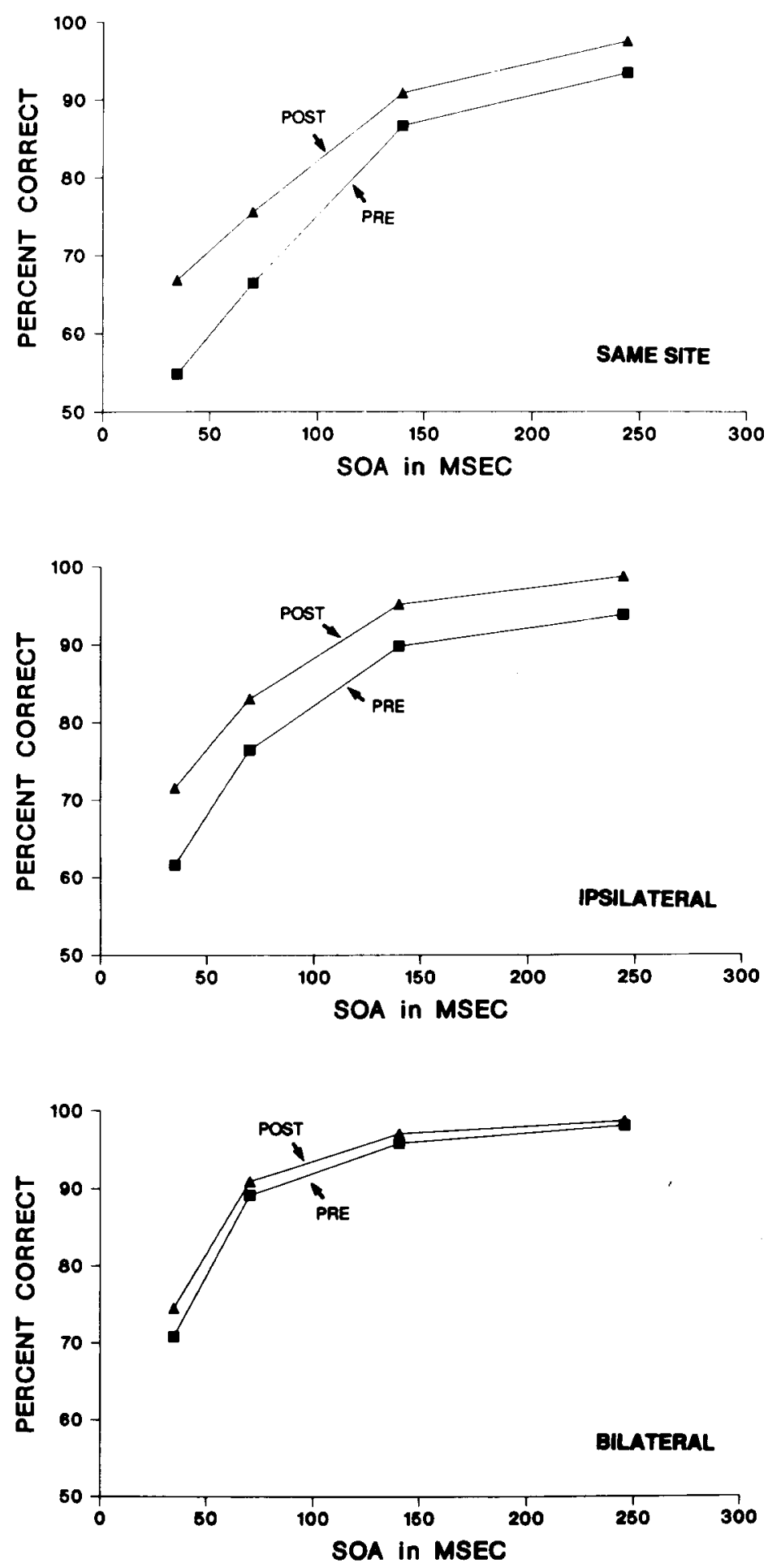

Figure 7. Percentage of correct responses in judging temporal order as a function of SOA. Similar to Figure 6, with the exception that the subjects were informed which two of the four patterns were to be presented. "Post" refers to this postinstruction condition. The functions marked "Pre" refer to the preinstruction conditions and are replotted from Figure 6. 


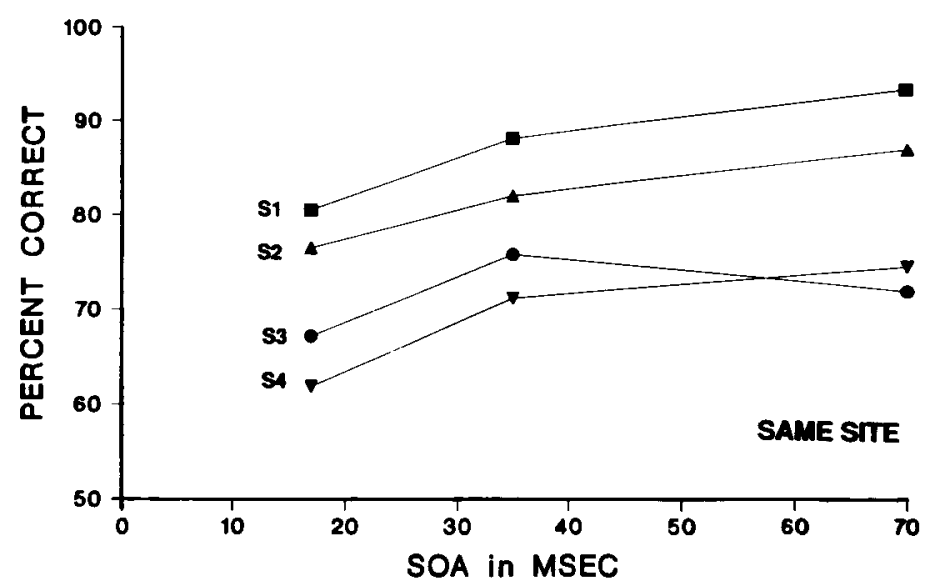

Figure 8. Percentage of correct responses in judging temporal order as a function of SOA. The subjects were tested on two of the patterns from Set A. Data from individual subjects are shown.

$74 \mathrm{msec}$, as compared to $12 \mathrm{msec}$ with the two patterns in Experiment 1. The conclusion, based on the results with Set $A$, is that same-site TOJs are particularly sensitive to the kind of patterns used.

With Set B, the subjects began testing with only two patterns and then went to three patterns and then four patterns. The threshold results are shown in Table 1 . The results support the conclusion from Set A patterns that the nature of the patterns had a large effect on same-site performance and less of an effect on separate-site performance. The same-site thresholds dropped to about a third of those seen with Set A (more comparable to those seen in Experiment 1), whereas the separate-site thresholds had, if anything, increased. The increase may be due to the lack of experience of the particular subjects. Increasing the number of patterns from two to three produced an increase in the same-site threshold, but no further increase in threshold is seen when the number of patterns was increased to four. As the number of patterns increased from three to four, the number of possible composites increased from 6 to 12 . The fact that a substantial increase in the number of composite patterns produced no increase in threshold suggests that subjects need not learn the shapes of composite patterns to judge temporal order in the same-site condition. The results in Table 1 also show that the same-site advantage was not limited to situations in which only a few patterns were to be judged.

At present, we have no clear indication of what it is about the patterns in Set $A$ that lead to such poor samesite performance. There are a number of differences be-

Table 1

TOJ Thresholds for Set B Patterns

\begin{tabular}{cccc}
\hline \multirow{2}{*}{$\begin{array}{c}\text { Number of Patterns } \\
\text { in Set }\end{array}$} & \multicolumn{3}{c}{ Threshold (in msec) } \\
\cline { 2 - 4 } & Same-Site & Ipsilateral & Bilateral \\
\hline 2 & 16 & 96 & 87 \\
3 & 28 & 110 & 82 \\
4 & 27 & 99 & 79 \\
\hline
\end{tabular}

tween the patterns in Set $A$ and the patterns that led to good same-site performance (Set B and the two patterns used in Experiment 1). Unlike pairs drawn from the other sets, pairs of patterns drawn from Set $A$, if presented simultaneously, or if presented at brief SOAs to form composite patterns, do not cross one another, and several pairs fail even to intersect. In addition, the Set B patterns and the patterns used in Experiment 1 cross one another at approximately the same location on the fingerpad. With these sets of patterns, the subjects could focus their attention on the same location on the fingerpad from trial to trial and could attempt to use cues that might be generated at the intersection of the two patterns. In Experiment 3, we examined one possible cue that subjects might use, relative intensity; however, several sets of patterns varying in different ways would have to be constructed to be certain what spatial features aid or interfere with TOJs.

\section{EXPERIMENT 3}

Experiments 1 and 2 showed that various stimulus manipulations affect TOJs for patterns delivered to the same location differently than for patterns delivered to separate locations. Although the subjects reported composite patterns only in the same-site condition, increasing the number of patterns, and thus the number of composites, did not necessarily produce poorer same-site performance (Experiment 2, Table 1). If not the shape of composite patterns, what cues are available in the samesite condition that are unavailable in the separate-site conditions? Relative intensity has already been mentioned as a possible cue. The subjects reported that the trailing stimulus felt more intense, and clearer, than the leading stimulus, an observation previously reported by Evans (1987b). Introducing an intensity imbalance between the two patterns might be expected to affect same-site judgments in the following way: When a more intense stimulus is presented first, subjects should respond incorrectly 
on a large proportion of trials. When a more intense stimulus is presented second, subjects should respond correctly on a large proportion of trials. In the latter case, the bias toward responding with the less intensely perceived stimulus will produce correct responses.

For patterns delivered to separate sites, the effect that an intensity imbalance might have is less clear. As noted before, the subjects did report that, in separate-site conditions, the first stimulus seemed to be more intense than the second. Previous studies in which TOJs were based just on location suggest that the more intense stimulus is selected as occurring first. Sherrick (1970) observed that when subjects were presented with an electrical and a mechanical stimulus to separate sites, the electrical stimulus felt much stronger than the mechanical stimulus at brief SOAs. Furthermore, when the two stimuli were presented at the same time, Sherrick's subjects reported the electrical stimulus to be first on $80 \%$ of the trials. With two mechanical stimuli, the subjects generally responded that the more intense stimulus was first, although this depended to some extent upon the location of the stimuli. These observations suggest that presenting stimuli of unequal intensity should alter TOJs and, furthermore, that the intensity imbalance might affect same-site and separate-site judgments differentially. In Experiment 3, we examined the effect of intensity manipulations on same-site and separate-site performance when subjects judged which one of two patterns was presented first.

\section{Method}

Subjects. One group of subjects was tested in the same-site condition. These subjects were also tested in separate-site conditions. However, because of an error in stimulus generation in the separatesite conditions, the separate-site data were discarded, and a second group of subjects was tested in the separate-site conditions.

Procedure. The major difference between the procedures in Experiment 3 and Experiment 1 was the intensity at which the pat- terns were presented. In Experiment 1, both members of the pair of patterns were set at the same level of intensity and that level was not changed. In Experiment 3, both the intensity level and the intensity relationship between members of the pairs of patterns were changed. On half of the trials, both patterns were presented at the same intensity; on the other half of the trials, the patterns were presented at unequal intensities. The intensity of each pattern was manipulated by adjusting the voltage to the pins of the tactile array. Two intensity levels were tested. The lower intensity was achieved by imposing $21 \mathrm{~V}$ on each pin, and the higher intensity was achieved by imposing $45 \mathrm{~V}$ on each pin. Using a magnitude-estimation procedure, subjects judged that patterns presented at $45 \mathrm{~V}$ were approximately 1.8 times more intense than patterns presented at $21 \mathrm{~V}$.

Within a block of trials, there were four different types of trials: both members of a pair of patterns could be at the same high (45V) or the same low ( $21 \mathrm{~V})$ intensity, or the members of a pair of pattems could be set at unequal intensity with the more intense stimulus first (high-low) or with the less intense stimulus first (low-high). The subjects were told that the intensity of the patterns would vary. The probability of occurrence of the four types of trials was equal (.25). Trials were presented in random order.

\section{Results and Discussion}

The results from the 5 subjects tested in the same-site condition are shown in Figure 9. For the equal-intensity trials, there was no difference in performance on the highequal and low-equal trials. These results were combined in Figure 9 and each point represents a total of 960 trials. For the unequal-intensity trials, the order of the intensity imbalance made a difference and these functions are shown separately. Each point represents a total of 480 trials. The effect of the intensity imbalance can be most clearly seen at the 17-msec SOA. On low-high trials, the correct response was that the less intense stimulus was first, and the subjects' responses were 93\% correct. On high-low trials, the correct response was that the more intense stimulus was first, but here the subjects were only $60 \%$ correct. In other words, when the two stimuli were separated by the same SOA, $17 \mathrm{msec}$, changing the rela-

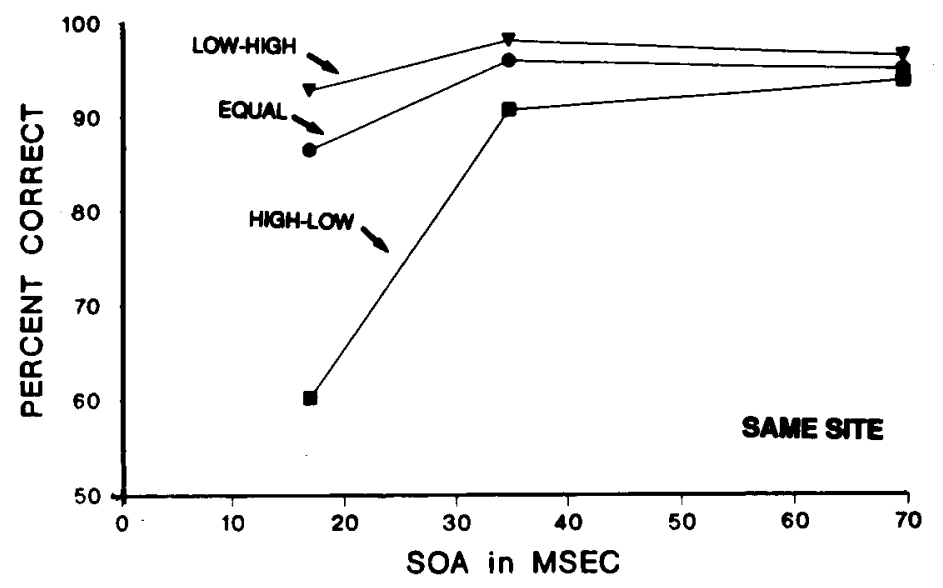

Figure 9. Percentage of correct responses in judging temporal order as a function of SOA. Stimuli were presented to the same site and were equal in intensity (equal), the more intense stimulus preceded the less intense stimulus (high-low), or the less intense stimulus preceded the more intense stimulus (low-high). The subjects had to judge which one of two patterns was presented first. 


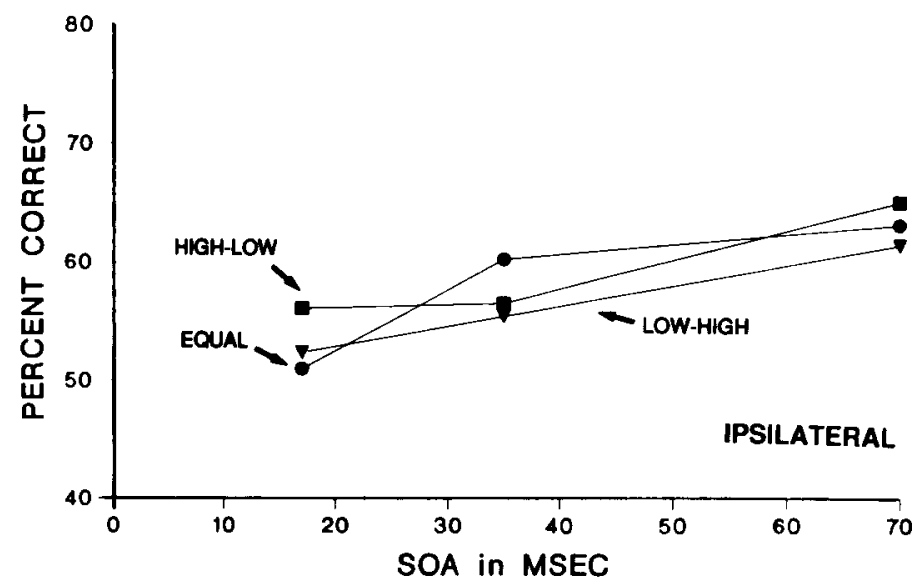

Figure 10. Percentage of correct responses in judging temporal order as a function of SOA. Similar to Figure 9, with stimuli presented to two sites on the same hand (ipsilaterally).

tive intensity of the two patterns produced a $33 \%$ shift in correct responding $(93 \%-60 \%)$.

A psychometric function was generated using the unequal-intensity, same-site data. The probability of reporting the more intense stimulus first was plotted as a function of SOA. For this function, the PSS was $11.5 \mathrm{msec}$. A stimulus 1.8 times as intense as a second stimulus would have to lead the second stimulus by about $11.5 \mathrm{msec}$ to be perceived as being simultaneous with it.

The effect of intensity imbalance in the same-site condition can be explained, at least at one level, in a rather straightforward way. The subjects had previously observed that the clearer and more intense of the two patterns was most often the second pattern presented; and, as noted before, each subject was informed that such an observation had been made by other subjects. Continuing to base their decisions on that earlier observation, the subjects would have been correct on a large proportion of the same-site trials. On half of the trials, the two pat- terns were equal in intensity. It is assumed that the second of the two patterns would be perceived as clearer and more intense. On half of the remaining trials, or $25 \%$ of all the trials, the direction of the intensity imbalance, low-high, would be consistent with the temporal order of the two patterns and would aid correct responding. It is only on the remaining $25 \%$ of the trials, high-low trials, that the intensity imbalance and temporal order would conflict. The intensity imbalance was insufficient to cause the subjects to always select the less intense stimulus as the leading stimulus but did, as noted before, shift their responding. Even if the intensity imbalance had been sufficient for the subjects' percentage of correct responses to drop to zero in the high-low condition, their overall percentage of correct responses would still be relatively high.

The results for the ipsilateral and bilateral measurements are shown in Figures 10 and 11. Each point for the equalintensity condition represents the performance of 4 subjects, 1,040 trials from each subject. For the unequal-

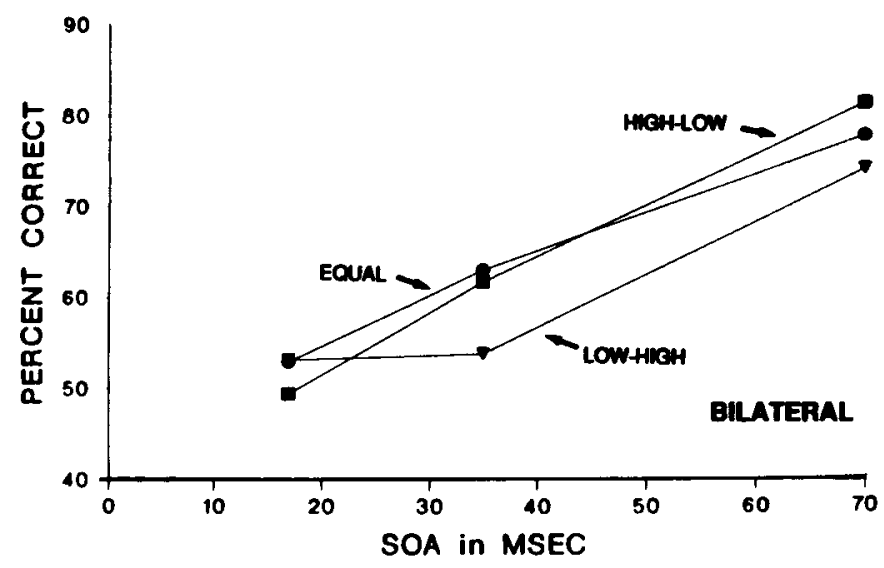

Figure 11. Percentage of correct responses in judging temporal order as a function of SOA. Similar to Figure 9, with stimuli presented to two sites on opposite hands (bilaterally). 
intensity conditions, each point represents $\mathbf{5 2 0}$ trials from 4 subjects. The separate-site results differ from the samesite results in several ways. First, the effect of intensity is much smaller in the separate-site conditions. Averaging across the three SOAs, the difference between high-low and low-high trials is $3 \%$ in the ipsilateral condition and $4 \%$ in the bilateral condition. Second, the effect of the intensity imbalance is in the opposite direction; that is, the more intense stimulus seemed to lead the less intense in the separate-site conditions. For the bilateral condition, presenting the more intense stimulus first (high-low) led to significantly better performance at the two longer SOAs ( $p<.01$ at $35 \mathrm{msec}$ and $p<.05$ at $70 \mathrm{msec}$ ). For the ipsilateral condition, there was no significant high-low advantage. As noted before, in TOJs involving location, the more intense stimulus seemed to lead the less intense. The slight bias favoring the more intense pattern in TOJs for the separate-site conditions is consistent with the view that subjects initially judge which location received a pattern first and then which pattern was presented to that location.

If a major cue to the same-site TOJs is the relative intensity of the two patterns, then is there something about the spatial configuration of the patterns in Set A in Experiment 2 that prevented this cue from being used? If there were differences in intensity between the patterns, relative intensity as a cue would be difficult to use. The number of stimulators in each pattern was the same, and thus, on this basis the perceived intensity of the patterns should be the same (Cholewiak, 1979). It is possible that the position of the patterns on the array might affect their perceived intensity. The two vertical patterns in Set A were presented at the edge of the array, where changes in innervation across the fingerpad might affect perceived intensity, or slight changes in the position of the subject's finger on the array might cause stimulators to lose contact with the fingerpad. Again, as discussed in Experiment 2, additional sets of patterns would have to be constructed to test these possibilities.

\section{GENERAL DISCUSSION}

The present study showed that when stimuli were delivered to separate sites on the skin, the subjects were more accurate in judging which location received a stimulus first than which pattern was presented first. This result is consistent with the view that subjects initially decide which location received a stimulus first and then which pattern was presented at that location. Separate-site temporal order thresholds for patterns would be greater than thresholds for location because of the additional step required to decide which pattern had been presented to a particular location. It is possible that location information and form information are processed independently of one another. In the visual modality, there is evidence of independence of location and pattern information (Krumhansl \& Thomas, 1976). One could certainly imagine situations in which subjects could report the location of a tactile pattern without being able to say what the pattern was. One could also imagine a situation in which a subject might correctly report the presence of a particular pattern and be unsure of its location. The latter situation might arise with multiple sites of stimulation and with stimuli being presented in close temporal proximity.

If temporal order for patterns can be judged separately from temporal order for location when stimuli are presented to different locations, then why should a greater temporal separation be required to judge correctly the order of two patterns than the order of two locations? One answer is that the increase in temporal separation may reflect a difference in the time required to process pattern information as compared to location information. If processing time for patterns is long relative to processing time for location, and is long relative to the SOAs needed for correct TOJs, and if such processing must be completed before the temporal order of the two patterns presented to separate sites can be judged, then greater thresholds for TOJs for patterns than for locations would be predicted.

The present results also have implications for the idea that separate fingers constitute separate channels for processing spatial patterns. Studies in which patterns were presented to one finger, two fingers on the same hand, or two fingers on opposite hands have generally shown much more interaction between patterns presented to the same finger (Craig, 1985a). The present results are clearly consistent with previous findings and offer additional support for the idea that separate fingers are separate channels for spatial information.

\section{REFERENCES}

Bliss, J. C., Katcher, M. H., Rogers, C. H., Shepard, R. P. (1970). Optical-to-tactile image conversion for the blind. IEEE Transactions on Man-Machine Systems, MMS-11, 58-64.

Chandler, J. P. (1970). STEPTT [QCPE, Program No. 66]. Bloomington, IN: Indiana University', Chemistry Department, Quantum Chemistry Program Exchange.

Cholewiak, R. W. (1979). Spatial factors in the perceived intensity of vibrotactile patterns. Sensory Processes, 3, 141-156.

Craig, J. C. (1982). Temporal integration of vibrotactile patterns. Perception \& Psychophysics, 32, 219-229.

CraIG, J. C. (1983). The role of onset in the perception of sequentially presented vibrotactile patterns. Perception \& Psychophysics, 34, $421-432$.

Craig, J. C. (1985a). Attending to two fingers: Two hands are better than one. Perception \& Psychophysics, 38, 496-511.

CraIG, J. C. (1985b). Tactile pattern perception and its perturbations. Journal of the Acoustical Society of America, 77, 238-246.

CraIg, J. C. (1989). Interference in localizing tactile stimuli. Perception \& Psychophysics, 45, 343-355.

Craig, J. C., \& Evans, P. M. (1987). Vibrotactile masking and the persistence of tactual features. Perception \& Psychophysics, 42, 309-317.

EVans, P. M. (1987a). Vibrotactile masking: Persistence, integration, and strength of representation. Unpublished doctoral dissertation, Indiana University, Bloomington.

Evans, P. M. (1987b). Vibrotactile masking: Temporal integration, persistence, and strengths of representations. Perception \& Psychophysics, 42, 515-525.

Evans, P. M., \& Craig, J. C. (1986). Temporal integration and vibro- 
tactile backward masking. Joumal of Experimental Psychology: Human Perception \& Performance, 12, 160-168.

Gengel, R. W., \& HiRSH, I. J. (1970). Temporal order: The effect of single versus repeated presentations, practice, and verbal feedback. Perception \& Psychophysics, 7, 209-211.

HilL, J. W. (1971). Processing of tactual and visual point stimuli sequentially presented at high rates. Journal of Experimental Psychology, 88, 340-348.

HiLL, J. W., Buss, J. C. (1968). Perception of sequentially presented tactile point stimuli. Perception \& Psychophysics, 4, 289-295.

Hirsh, I. J., \& Sherrick, C. E. (1961). Perceived order in different sense modalities. Journal of Experimental Psychology, 62, 423-432.

Krumhansl, C. L., Thomas, E. A. C. (1976). Extracting identity and location information from briefly presented letter arrays. Perception \& Psychophysics, 20, 243-258.

Marks, L. E. , Girvin, J. P., O'KeEfe, M. D., Ning, P., Quest, D. O., ANTUNES, J. L., DOBeLle, W. H. (1982). Electrocutaneous stimulation: 3. The perception of temporal order. Perception \& Psychophysics, 32, 537-541.
Sherrick, C. E. (1968a). Bilateral apparent haptic movement. Perception \& Psychophysics, 4, 159-160.

SHERRICK, C. E. (1968b). Studies of apparent tactual movement. In D. R. Kenshalo (Ed.), The skin senses (pp. 331-344). Springfield, IL: Charles C. Thomas.

SHERrICK, C. E. (1970). Temporal ordering of events in haptic space. IEEE Transactions on Man-Machine Systems, MMS-11, 25-28.

SHERRICK, C. E., Rogers, R. (1966). Apparent haptic movement. Perception \& Psychophysics, 1, 175-180.

SterngerG, S., \& KNoll, R. L. (1973). The perception of temporal order: Fundamental issues and a general model. In S. Kornblum (Ed.), Attention and performance (Vol. 4, pp. 629-685). New York: Academic Press.

TAYLOR, B. (1978). Dimensional redundancy in the processing of vibrotactile temporal order. Unpublished doctoral dissertation, Princeton University, Princeton, NJ.

(Manuscript received July 1, 1988; revision accepted for publication July $11,1989$. 